\title{
CATALOGO CRITICO Y NOMINAL DEL GENERO DARGIDA WALKER 1856 (LEPIDOPTERA, NOCTUIDAE, HADENINAE)
}

\author{
CRITIC AND NOMINAL CATALOGUE OF THE GENUS DARGIDA WALKER \\ 1856 (LEPIDOPTERA, NOCTUIDAE, HADENINAE)
}

\author{
Marcela A. Rodríguez \& Andrés O. Angulo \\ Departamento de Zoología, Facultad de Ciencias Naturales y Oceanográficas, Universidad de Concepción. Casilla 160- \\ C, Concepción-Chile.E-mail: marcrodr@udec.cl; aangulo@udec.cl
}

\section{RESUMEN}

El presente catálogo consiste en la lista alfabética de los nombres de las especies pertenecientes al género Dargida Walker, 1856 y que fueron descritas antes del 1 de junio del 2003. Strigania Hampson, 1905 y Faronta Smith, 1908 son sinónimos de Dargida Walker, 1856 por comparación de las especies tipo de cada género. El tratamiento dedicado a cada taxon específico incluye su sinonímia confirmada por citas y una mención de la localidad tipo. Como resultado, se obtiene una lista de 53 especies asignadas al género Dargida Walker. Se entregan 14 nuevas combinaciones.

Palabras Claves: Dargida, Faronta, Strigania, Hadeninae, Noctuidae, Región Neotropical, Región Andino-Patagónica, nuevas combinaciones, nuevos registros.

\section{ABSTRACT}

This alphabetical list consists of the names of all species belong to Dargida Walker, 1856 genus that were described prior to 1 june 2003; Strigania Hampson, 1905 and Faronta Smith, 1908 are synonymies of Dargida Walker, 1856 by comparison with the type species of each genus. In the treatment of each specific taxon the synonymy by confirmation bibliographic is given and a mention of type locality. 53 species of Dargida Walker genus, are listed. The fourtheen new combinations are done.

Keywords: Dargida, Faronta, Strigania, Hadeninae, Noctuidae, Neotropical Region, Andean-Patagonian Región, new combinations, new records.

\section{INTRODUCCION}

Faronta albilinea (Hübner) y F. atrifera (Hampson) son especies que presentan importancia económica ya que sus estadios larvales se encuentran asociados a cereales y gramíneas (arroz, avena, cebada, trigo) (Angulo \& Weigert 1975). Angulo et al. (1999) establecen que Faronta Smith, 1908 es un sinónimo junior de Strigania Hampson, 1905.
Dargida y Faronta forman un grupo natural sensu Franclemont (1951) como miembros de uno de los grupos que el autor reúne y los separa de los otros géneros de hadeninos por la presencia, en la genitalia del macho, de espinas uniseriales en la corona de la valva; Además advierte que ambos géneros comparten especies muy homogéneas y que la genitalia tanto en machos como en hembras no muestra reales diferencias, pero sin embargo, su maculación 
alar difiere, siendo éste y la presencia de penachos los caracteres base para mantenerlos separados. Posteriormente, Forbes (1954), en una revisión del "grupo Leucania Ochsenheimer", advierte que el grupo "albilinea" del género Faronta Smith, que presenta corona simple, forma junto a Dargida una agrupación. Godfrey (1972) encuentra un único carácter que separa ambos géneros, basado en los estados larvales de Faronta y Dargida, donde la larva de Dargida presenta los bordes internos de la mandíbula paralelos, por lo tanto separados, y los de Faronta están interconectados. La comparación de los tipos de Strigania Hampson, 1905 con Dargida Walker, 1856 establecen la sinonimia entre ambos, quedando como género válido Dargida Walker, por principio de prioridad.

\section{MATERIALES Y METODOS}

Se examinaron ejemplares de las especies de los géneros Strigania Hmps., Dargida Walker y Faronta Smith, provenientes de las colecciones de los siguientes museos: Colecciones Científicas Universidad de Concepción (UCCC), Museo Británico de Historia Natural, Londres (BMNH), Smithsonian Institution, National Museum of Natural History, Washington (USNM) y Museo Ecuatoriano de Ciencias Naturales (MECN).

Se obtuvieron especímenes provenientes de recolecciones de terreno por medio de trampas fototrópicas de luz ultravioleta, en:

a) Laguna El Maule, Paso Internacional Pehuenche 2560 m.s.n.m. (35 58' 56" S - 70 23' 70" W), VII Región-Chile y b) Sector La Mina, control aduanero 930 m.s.n.m. (35 $57^{\prime} \mathrm{S}-71^{\circ}$ 23’ O), VII Región-Chile.

Para la identificación del material se utilizó la comparación con los tipos.

Se entrega la descripción original del género Dargida Walker, 1856, agregando las descripciones de las estructuras faltantes. La comparación de los tipos fue realizada sobre la base de: maculación alar; morfología externa; estructura de la armadura genital del macho y de la hembra.

Para la obtención de las estructuras internas se utilizó la técnica corriente de extracción sensu, Angulo \& Weigert (1977).

\section{RESULTADOS}

En el estudio de Angulo et al., 1999, en donde el género Strigania Hampson se sinonimiza con Faronta Smith, queda de manifiesto la cercanía con el género Dargida Walter, cuando se comparan las especies tipos de los géneros involucrados: Dargida grammivora Walker, Faronta aleada Smith y Strigania lithophilus (Butler); ellos muestran diferencias notables en maculación alar, sin embargo, los restantes caracteres externos las sugieren como especies congenéricas, luego en el análisis y comparación de las estructuras genitales de los machos de estos géneros más bien las especies muestran gran cantidad de caracteres compartidos albergados en formas extremas. Por otra parte, la genitalia de la hembra muestra caracteres compartidos al punto de ser estas estructuras prácticamente indistinguibles entre las tres especies estudiadas. Los resultados de esta comparación revelaron que las especies tipos de los géneros problema comparten caracteres que imposibilitan su separación morfológica en este nivel taxonómico, por lo tanto, Strigania Hampson, 1905 y Faronta Smith, 1908 son sinónimos junior de Dargida Walker, 1856, el cual es válido por principio de prioridad.

Los caracteres genitales principales que reúnen a estos géneros están agrupados en una tabla comparativa (Tabla I).

El análisis de estos caracteres nos permite establecer la diagnosis genérica basada en las estructuras genitales de los machos y hembras del género Dargida Walker. 
Tabla I: Comparación entre los caracteres genitales de los géneros Dargida Walker 1856; Strigania Hampson 1905 y Faronta Smith 1908

TABLE I. Comparison between the genital characters of the genus Dargida Walker 1856; Strigania Hampson 1905 y Faronta Smith 1908

\begin{tabular}{|c|c|c|c|}
\hline CARACTERES & $\begin{array}{l}\text { Strigania Hampson } \\
\text { S. lithophillus (But.) } \\
\text { (Figs. } 2,6,8,11 \text { ) }\end{array}$ & $\begin{array}{c}\text { Dargida Walker } \\
\text { D. grammivora Walk. } \\
\text { (Figs. } 1,4,7,10)\end{array}$ & $\begin{array}{l}\text { Faronta } \text { Smith } \\
\text { F. aleada } \text { Smith } \\
\text { (Figs. } 3,5,9,12)\end{array}$ \\
\hline \multicolumn{4}{|l|}{ MACHO: } \\
\hline Uncus & - Espatulado & - Espatulado & - Espatulado \\
\hline $\begin{array}{l}\text { Engrosamiento basal del } \\
\text { uncus }\end{array}$ & - Presente & - Presente & - Presente \\
\hline Yuxta & - Ancoriforme & - Ancoriforme & - Ancoriforme \\
\hline Sacus & - Romo & - Romo algo aguzado & - Romo algo aguzado \\
\hline $\begin{array}{l}\text { Posición cuello hadenino y } \\
\text { complejo del clásper }\end{array}$ & - Tercio apical de la valva & - Tercio apical de la valva & - Tercio apical de la valva \\
\hline Corona & - Uniseriada & - Uniseriada & - Uniseriada \\
\hline - Expansión del cucullus & $\begin{array}{l}\text { - Asimétrico, porción } \\
\text { ventral con más desarrollo }\end{array}$ & $\begin{array}{l}\text { - Asimétrico, porción } \\
\text { ventral con más desarrollo }\end{array}$ & $\begin{array}{l}\text { - Asimétrico, porción } \\
\text { ventral con más desarrollo }\end{array}$ \\
\hline Clásper & - Vestigial & - Vestigial & - Vestigial \\
\hline Dígitus & $\begin{array}{l}\text { - Laminar, de forma } \\
\text { subcuadrangular con un } \\
\text { proceso dorsal saliente de la } \\
\text { valva }\end{array}$ & $\begin{array}{l}\text { - Laminar, de forma } \\
\text { subcuadrangular sin proceso } \\
\text { saliente, pero con vestigio } \\
\text { de éste }\end{array}$ & $\begin{array}{l}\text { - Laminar, de forma } \\
\text { subtriangular, sin } \\
\text { proyección dorsal }\end{array}$ \\
\hline Ampulla & $\begin{array}{l}\text { - Bien desarrollada c/ } \\
\text { crenulado central }\end{array}$ & $\begin{array}{l}\text { - Bien desarrollada s/ } \\
\text { crenulado central }\end{array}$ & $\begin{array}{l}\text { - Bien desarrollada c/ } \\
\text { crenulado central }\end{array}$ \\
\hline Aedeagus & $\begin{array}{l}\text { - Con corrida de } 4 \text { a } 7 \\
\text { espinas fuertes en la base de } \\
\text { la vésica, otras dispersas y } \\
\text { microespinas centrales }\end{array}$ & $\begin{array}{l}\text { - Sin corrida de espinas en } \\
\text { base de la vésica, con } \\
\text { espinas gruesas y largas en } \\
\text { el centro de la vésica }\end{array}$ & $\begin{array}{l}\text { - Con corrida de espinas en } \\
\text { la base de la vésica y con } \\
\text { microespinas centrales }\end{array}$ \\
\hline \multicolumn{4}{|l|}{ HEMBRA: } \\
\hline Bursa copulatrix & - Bisacular & - Bisacular & - Bisacular \\
\hline Disposición de los signa. & $\begin{array}{l}\text { - En hilera de la base al polo } \\
\text { del corpus }\end{array}$ & $\begin{array}{l}\text { - En hilera de la base al polo } \\
\text { del corpus }\end{array}$ & $\begin{array}{l}\text { - En hilera de la base al polo } \\
\text { del corpus }\end{array}$ \\
\hline Forma de signa & $\begin{array}{l}\text { - Doble placa de } \\
\text { microespinas }\end{array}$ & $\begin{array}{l}\text { - Doble placa de } \\
\text { microespinas }\end{array}$ & $\begin{array}{l}\text { - Doble placa de } \\
\text { microespinas }\end{array}$ \\
\hline $\begin{array}{l}\text { Tamaño de cervix con } \\
\text { relación al corpus bursae }\end{array}$ & - Pequeño & - Pequeño & - Pequeño \\
\hline $\begin{array}{l}\text { Apófisis de los lóbulos del } \\
\text { ovipositor }\end{array}$ & - Sub-iguales & - Sub-iguales & - Sub-iguales \\
\hline $\begin{array}{l}\text { Forma de los lóbulos del } \\
\text { ovipositor }\end{array}$ & - Sub-cuadrangulares & - Sub-cuadrangulares & - Sub-cuadrangulares \\
\hline - Ductus bursae & - Con estriación & - Sin estriación & - Con estriación \\
\hline
\end{tabular}




\section{DARGIDA Walker, 1856}

Dargida Walker, 1856: 206

Eupsephopaectes Grote, 1873: 137, sensu Poole 1989: 307

Strigania Hampson, 1905: 466 Nuevo Sinónimo. Faronta Smith, 1908: 106. Angulo et. al. 1999: 68

Pseudoleucania Mc Dunnough, 1937a. Nye 1975:408

Protoleucania Mc Dunnough $1937 \mathrm{~b}$. Franclemont, 1951: 63; Nye 1975:408.

Adulto: (Fig. 1) "Probóscide completamente desarrollada; palpo oblicuo, el 2do segmento al frente con flecos de largos pelos, el 3er segmento corto, dirigido hacia adelante; frente lisa; ojos, redondos cubiertos por largas cilias; cabeza y tórax cubierto con toscos pelos y escamas; tibia con margen cubierta por pelos, moderadamente; abdomen con algunos pelos en la base del dorso, pero sin crestas. Ala anterior con el termen oblicuo y curvado; venas 4 y 5 desde cerca de la areola; 11 desde la celda. Ala posterior con venas 3, 4 desde el ángulo de la celda; 5 en desuso desde el medio de la celda discal; 6, 7 desde el ángulo superior; 8 anastomosada con la celda cerca de la base" (Walker 1856).

\section{CARACTERes GenÉricos genitales:}

Genitalia del macho: (Figs.4-6) uncus espatulado, valva con cuello hadenino muy pronunciado; corona uniserial provista por espinas fuertes, cucullus con expansión ventral más desarrollada que la dorsal; clásper sin desarrollo; ampulla y dígitus bien desarrollados, dígitus con o sin una proyección dorsal desarrollada; complejo del clásper ubicado en el tercio distal; yuxta ancoriforme. Aedeagus (Figs.7-9) con vésica provista por un peine de espinas o microespinas central; con apófisis dorsal de la vésica armada por cordón de espinas bajas y gruesas o inerme.

Genitalia de la hembra: (Figs.10-12) apófisis anteriores y posteriores subiguales en tamaño; bursa copulatrix bisacular, corpus bursae de mayor tamaño que el cervix bursae, el corpus bursae suboval, provisto de signa en número variable con forma de doble placa de microespinas ubicadas en hilera y dispuestas equidistantes una de la otra desde el polo a la base del corpus; cervix sacular alargado de disposición variable; ductus bursae liso con estriaciones en la porción superior; lóbulos del ovipositor subcuadrangulares en vista lateral.

Material examinado: $2 \sigma^{\top}$ Ecuador-Imbabura, 44-1989, Ruales: coll, A. O. Angulo det. (UCCC); 1 ㅇ Ecuador-Imbabura, 5-4-1988, trampas: coll, A. O. Angulo det. (UCCC); $10^{*}$ I Región, Iquique, Parca (Juan Cayo) 1-1952, A. O. Angulo: det (UCCC); 1 ㅇ Huanuco, 6-4-1984, A. O. Angulo: det (UCCC); 1 o Huanuco, 21-11-1982, A. O. Angulo: det (UCCC); 1 Holotype. Chili, Valparaiso, 1950, J. J. Walker: coll 93-162, Meliana atrifera tipo Hmps. (BMNH). $10^{*}$ Syntype. Chili, 1954, Edmond: coll 82-107, Noctuidae Brit. Mus. Slide $\mathrm{N}^{\circ}$ 9894, Strigania lithophilus Butler (BMNH). $20^{\star}$ y $10^{\star}$ Cumtypus cumparatum M. G. Pogue, 1999. Padre Island Nat. Seashore, Texas 24.IV.76, A \& M.E. Blanchard (UCCC). 1 i Cumtypus cumparatum M. G. Pogue, 1999. Padre Island Nat. Seashore, Texas 19.VII.76, A \& M.E. Blanchard (UCCC).

\section{CATALOGO CRITICO NOMINAL DEL GENERO DARGIDA WALKER 1856}

1. Dargida acanthus (Herrich-Schäffer) Noctua acanthus Herrich-Schäffer 1869: 4 Dargida acanthus (Herrich-Schäffer). Poole 1989: 307

Localidad tipo: Venezuela.

\section{Dargida acca (Herrich-Schäffer)}

Noctua acca Herrich-Schäffer, 1869: 4

Dargida acca (Herrich-Schäffer). Poole 1989: 307

Localidad tipo: Venezuela

\section{Dargida albifluviata (Druce)}

Mamestra albifluviata Druce 1905: 372

Dargida albifluviata (Druce). Poole 1989: 372

Localidad tipo: Colombia

4. Dargida albilinea (Hübner) n. comb. Leucania albilinea Hübner, 1827 (fide Poole 1989: 447) 
Gayana 69(1), 2005
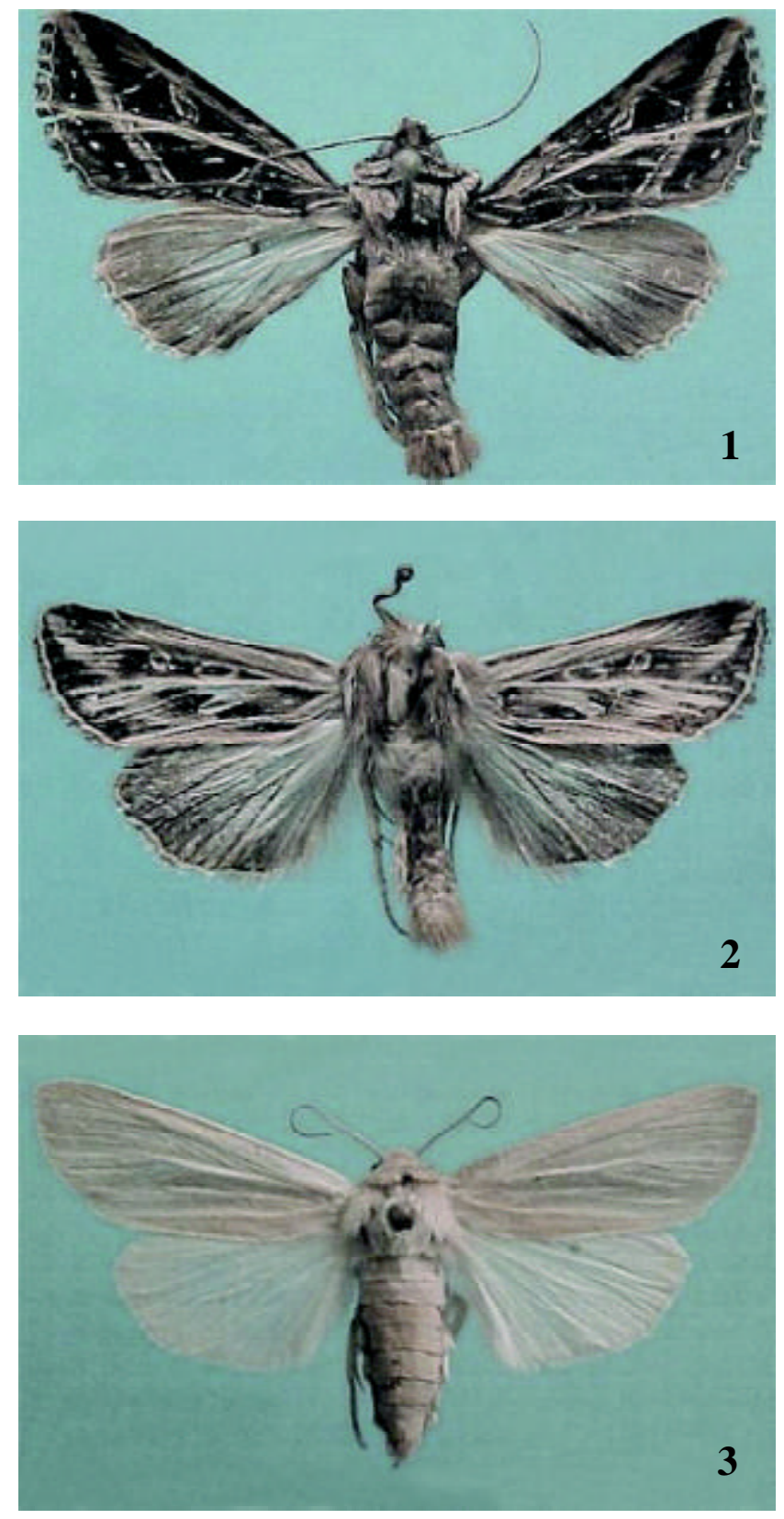

Figuras 1-3: Adulto de: Fig. 1. Dargida grammivora Walker; Fig. 2. Dargida lithophilus (Butler); Fig. 3. Dargida aleada (Smith). Escala= $1 \mathrm{~mm}$.

Figures 1-3: Adult of Fig. 1. Dargida grammivora Walker; Fig. 2. Dargida lithophilus (Butler); Fig. 3. Dargida aleada (Smith). Scale $=1 \mathrm{~mm}$. 


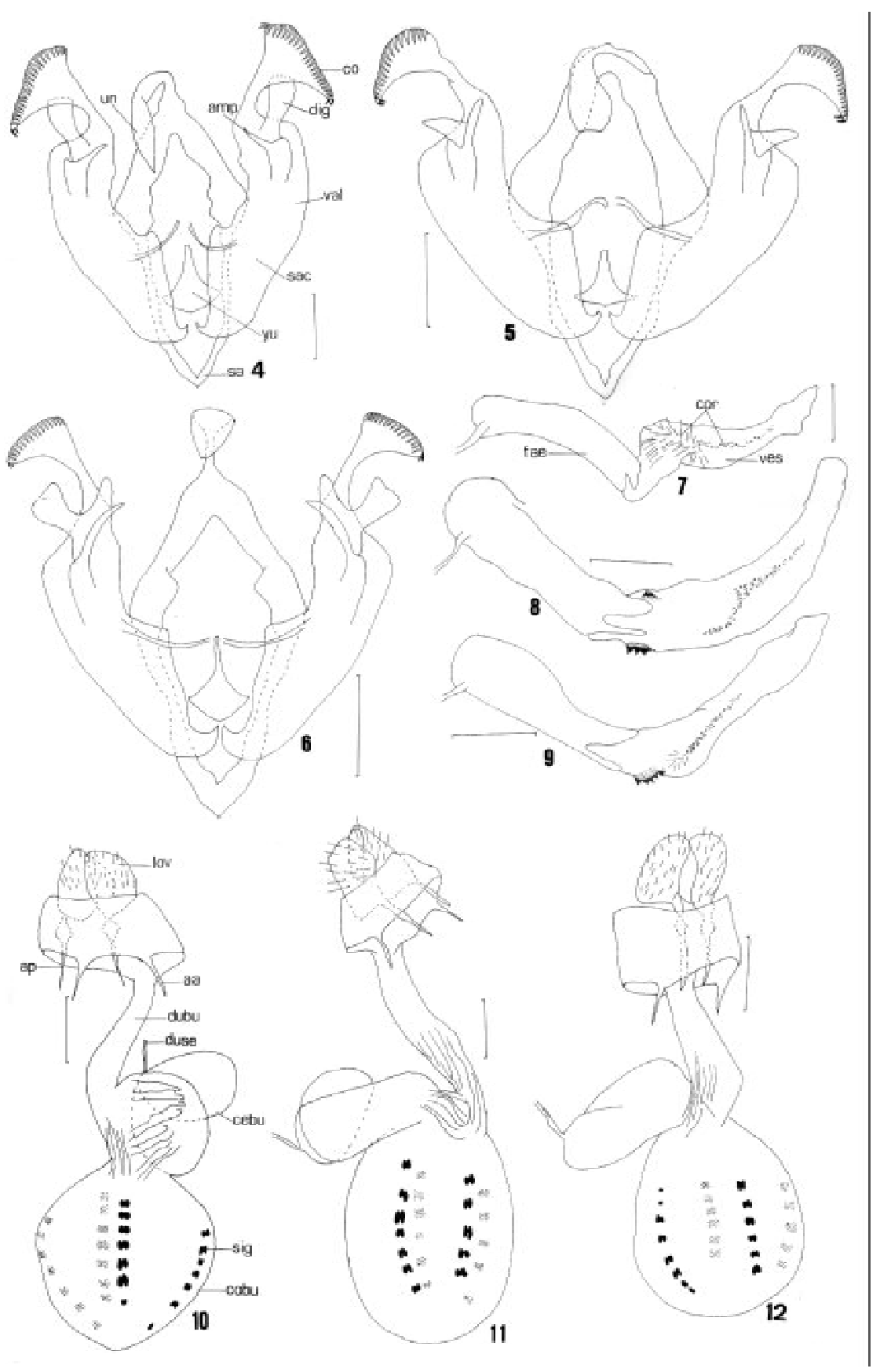

Figuras 4-12: Valvas del macho de: Fig. 4. D. grammivora Walker; Fig. 5. D. aleada (Smith); Fig. 6. D. lithophilus (Butler) (amp: ampulla; co: corona; dig: digitus; sa: sacus; sac: sacculus; un: uncus; val: valva; yu: yuxta). Escala = 1mm. Aedeago de: Fig. 7. D. grammivora Walker (cor: cornuti; fae: funda del aedeago; ves: vésica); FIG. 8. D. aleada (Smith); Fig. 9. D. lithophilus (Butler). Escala =1mm; Genitalia de la hembra de: FIG. 10. D. grammivora Walker (aa: apófisis anteriores; ap: apófisis posteriores; cebu: cervix bursae; cobu: corpus bursae; dubu; ductus bursae; duse: ductus seminalis; lov: lóbulos del ovipositor; sig: signa); FIG. 11. D. aleada (Smith); Fig. 12. D. lithophilus (Butler). Escala= $1 \mathrm{~mm}$.

Figures 4-12: Valves of the male of : Fig. 4. D. grammivora Walker; FIg. 5. D. aleada (Smith); Fig. 6. D. lithophilus (Butler) (amp: ampulla; co: corona; dig: digitus; sa: saccus; sac: sacculus; un: uncus; val: valva; yu: yuxta). Scale $=1 \mathrm{~mm}$.

Aedeago of: Fig.7. D. gramivora Walker (cor: cornuti; fae: funda del aedeago; ves: vésica); FIG. 8. D. aleada (Smith); Fig.9. D. lithophilus (Butler). Scale $=1 \mathrm{~mm}$; Genitalia female of the: Fig.10. D. grammivora Walker (aa: apófisis anteriores; ap: apófisis posteriores; cebu: cervix bursae; cobu: corpus bursae; dubu; ductus bursae; duse: ductus seminalis; lov: lóbulos del ovipositor; sig: signa); Fig.11. D. aleada (Smith); FIg.12. D. lithophilus (Butler). Scale =1 mm. 
Leucania diffusa Walker 1856 (fide Parra \& Angulo 1988)

Leucania moderata Walker 1856 (fide Parra \& Angulo 1988)

Leucania chilensis Butler 1882: 115 (fide Parra \& Angulo 1988)

Meliana albilinea (Hübner) Hampson 1905, p.583. Bullock 1943, p.1.

Faronta albilinea (Hübner). Casella y Moratorio 1971, p. 261; Angulo \& Weigert 1975, P.40; Parra et al. 1986, p.86.

Strigania albilinea (Hübner), Angulo et al. 1999: 68 Localidad tipo: Chile: Las Zorras (L. chilensis). Argentina: Buenos Aires (F. albilinea). Localidad desconocida (L. moderata).

\section{Dargida albistriga (Druce)}

Miselia albistriga Druce 1908: 289

Dargida albistriga (Druce). Poole 1989: 307

Localidad tipo: Perú: Puno, Santo Domingo

6. Dargida albomarginata (Druce)

Calaena albomarginata Druce in Godman \& Salvin 1898: 478.

Dargida albomarginata (Druce). Poole 1989: 307. Localidad tipo: Costa Rica: Candelaria.

\section{Dargida albostriata (Druce)}

Polia albostriata Druce 1911: 719

Dargida albostriata (Druce). Poole 1989: 307

Localidad tipo: Perú: Acopampa

8. Dargida aleada (Smith) n. comb.

Faronta aleada Smith 1908: 107

Strigania aleada (Draudt). Angulo et al. 1999

Localidad tipo: USA: Texas, Brazos.

9. Dargida amoena (Draudt) n. comb.

Neleucania amoena Draudt in Seitz 1924: 170

Localidad tipo: Bolivia: Songo.

10. Dargida calographa (Maassen)

Mamestra calographa Maassen in Weymer \& Maassen, 1890: 140

Dargida calographa (Maassen), Poole, 1989: 307.

Localidad tipo: Ecuador: Antisana, Cimaronas

11. Dargida clavifera (Hampson)

Hadena clavifera Hampson, 1909: 379

Dargida clavifera (Hampson). Poole, 1989: 307
Localidad tipo: Perú: Puno, Agualani.

12. Dargida clavisigna (Hampson)

Hadena clavisigna Hampson, 1913:593

Dargida clavisigna (Hampson). Poole, 1989:307

Localidad tipo: Perú: Acopampa

\section{Dargida confundibilis (Köhler)}

Faronta confundibilis Köhler, 1973: 21

Dargida confundibilis (Köhler). Angulo \& Olivares 1999 b.

Localidad tipo: Argentina: Neuquén, Collon-Cura.

14. Dargida demerodes (Dyar) n. comb.

Scriptania demerodes Dyar, 1920: 164; Poole, 1989: 902.

Strigania demerodes (Dyar). Rodríguez et al., 2001: 325.

Localidad tipo: México: Ciudad de México.

15. Dargida diffusa (Walker) n. comb.

Leucania diffusa Walker, 1856: 94.Poole, 1989: 447

Leucania moderata Walker, 1856. Poole, 1989: 448

Leucania harveyi Grote, 1873: 9. Poole, 1989: 447

Leucania limitata Smith, 1902: 187. Poole, 1989: 447

Leucania neptis Smith, 1902: 188. Poole, 1989: 448

Leucania obscurior Smith, 1902: 185. Poole, 1989: 448

Strigania diffusa (Walker). Angulo \& Olivares 1999

Localidad tipo: Canadá: Nova Scotia (L. diffusa); Manitoba - Cartwright (L. obscurior); USA: New York-Buffalo (L. harveyi); Colorado (L. neptis); Texas (L. limitata). Localidad desconocida ( $L$. moderata).

16. Dargida dissentanea (Draudt)

Nephelistis dissentanea Draudt in Seitz, 1924: 119 Dargida dissentanea (Draudt) Poole, 1989: 307 Localidad tipo: Bolivia: Cuesta von Cillutincara.

17. Dargida disticta (Druce) n. comb. Meliana disticta Druce, 1908: 295

Faronta disticta (Druce) Poole, 1989: 448 Strigania disticta (Druce) Angulo et al. 1999 Localidad tipo: Perú: Puno, Carabaya, Quinton. 
18. Dargida egregia (Draudt)

Polia egregia Draudt in Seitz, 1924: 104

Dargida egregia (Draudt). Poole, 1989: 307

Localidad tipo: Colombia: Tolima, Paso Quindiu.

19. Dargida eleistis (Druce) [emend.]

Hadena elaeistis Druce, 1905: 373. Hampson, 1905: 606

Dargida elaeistis (Druce). Poole, 1989: 307

Localidad tipo: Perú: Huancabamba.

20. Dargida eugrapha (Hampson)

Hadena eugrapha Hampson, 1909: 378

Dargida eugrapha (Hampson) Poole, 1989: 307

Localidad tipo: Perú: Limbani.

21. Dargida exoul (Walker) n. comb.

Leucania exoul Walker, 1856: 109

Faronta exoul (Walker). Poole, 1989: 448

Leucania tenebrifera Walker, 1856: 113; Poole, 1989:448

Strigania exoul (Walker). Angulo et al. 1999

Localidad tipo: Tristan da Cunha.

22. Dargida faeculenta (Draudt)

Poliodestra faeculenta Draudt in Seitz, 1924: 153

Dargida faeculenta (Draudt). Poole, 1989: 307

Localidad tipo: Colombia: Tolima, Paso Quindiu.

\section{Dargida fluminalis (Dognin)}

Nephelistis fluminalis Dognin, 1911: 16

Dargida fluminalis (Dognin). Poole, 1989: 307

Localidad tipo: Colombia: Tolima, Páramo de Quindiu.

24. Dargida ganeo (Draudt)

Polia ganeo Draudt, 1924: 107

Dargida ganeo (Draudt). Poole, 1989: 307

Localidad tipo: Bolivia: Cuesta von Cillutincara.

25. Dargida graminea Schaus

Dargida graminea Schaus, 1894: 231. Poole, 1989: 307

Hadena graminea (Schaus) Hampson, 1905: 209

Localidad tipo: Brasil: Paraná, Castro.

26. Dargida grammivora Walker

Dargida grammivora Walker, 1856: 202. Poole, 1989: 307

Hadena grammivora (Walker). Hampson, 1905: 210.
Localidad tipo: Venezuela. Nuevo registro: Chile (Iquique)

Nuevo registro: Ecuador, Chile. Perú

27. Dargida gumia (Draudt)

Polia gumia Draudt, 1924: 106

Dargida gumia (Draudt) Poole, 1989: 307

Localidad tipo: Bolivia: Cuesta von Cillutincara.

28. Dargida hieroglyphera (Maassen)

Moma hieroglyphera Maassen, 1890: 137, sensu Poole, 1989: 307

Dargida hieroglyphera (Maassen). Poole, 1989: 307. Localidad tipo: Ecuador: Antisana.

29. Dargida imitata (Maassen)

Mamestra imitata Maassen, 1890: 137, sensu Poole, 1989: 307

Dargida imitata (Maassen). Poole, 1989: 307

Localidad tipo: Ecuador: Collanes am Altar.

30. Dargida jucunda (Maassen) n. comb. Acronycta jucunda Maassen, 1890: 141

Strigania jucunda (Maassen) Draudt in Seitz, 1927: 162

Dargida jucunda (Maassen) Poole, 1989: 307, sensu Poole, 1989: 307

Localidad tipo: Colombia: Bordoncillo.

31. Dargida jucundissima (Zerny)

Dryobota jucundissima Zerny, 1916: 181

Dargida jucundissima (Zerny) Poole, 1989: 307

Localidad tipo: Brasil: Sao Paulo, Ypanema.

32. Dargida leucoceps (Hampson)

Hadena leucoceps Hampson, 1913: 592

Dargida leucoceps (Hampson). Poole, 1989: 307

Localidad tipo: Perú: Acopampa.

33. Dargida lithophilus (Butler) n. comb.

Heliophobus lithophilus, Butler, 1882: 119. Tipo visto.

Strigania lithophilus (Butler, 1882). Hampson, 1905: 466

Neleucania multistria Köhler, 1947: 1902

Faronta multistria (Köhler). Poole, 1989: 448; Angulo \& Olivares, 1999: 57

Meliana atrifera, Hampson, 1905: 580. Tipo visto. Faronta atrifera (Hampson, 1905). Parra \& Angulo, 1988: 263 
Leucania atrifera (Hampson, 1905). Poole, 1989: 577. Localidad tipo: Argentina: Tucumán (F. multistria); Siambon (M. atrifera). Chile: Valparaíso $(H$. lithophilus).

\section{Dargida melanoleuca (Druce)}

Miselia melanoleuca Druce, 1908: 292

Dargida melanoleuca (Druce). Poole, 1989: 307

Localidad tipo: Perú: Puno, Carabaya, Oconeque.

35. Dargida meridionalis (Hampson)

Hadena meridionalis Hampson 1905: 211

Dargida meridionalis (Hampson). Poole, 1989: 307

Localidad tipo: Brasil: Río de Janeiro.

36. Dargida mesotoma (Hampson)

Hadena mesotoma Hampson, 1909: 378

Hadena boliviana Köhler, 1968: 11 sensu Poole, 1989:307

Dargida mesotoma (Hampson). Poole, 1989: 307

Strigania boliviana (Köhler). Angulo \& Olivares, 1999.

Localidad tipo: Perú: Puno, Agualani ( $H$. mesotoma). Bolivia: Cuticucho, Río Songo $(H$. boliviana).

37. Dargida napali (Köhler) n. comb. Protoleucania napali Köhler, 1959: 38 Faronta napali (Köhler). Poole, 1989: 448 Strigania napali (Köhler). Angulo et al., 1999 Localidad tipo: Argentina: Buenos Aires, Tandil, Villa Gassel.

\section{Dargida nectaristis (Draudt)}

Hadena nectaristis Draudt, 1924: 114

Dargida nectaristis (Draudt), Poole, 1989: 307

Localidad tipo: Colombia: Monte Tolima.

39. Dargida oenistis (Druce)

Hadena oenistis Druce, 1905: 373; Hampson, 1905 : 607

Dargida oenistis (Druce). Poole, 1989: 307

Localidad tipo: Perú: Huancabamba.

40. Dargida permira (Draudt)

Strigania permira Draudt, in Seitz, 1924: 163; Poole, 1989: 936

Strigania permira Köhler secundum Angulo \& Olivares, 1999: 57

Localidad tipo: Perú: Madre de Dios; Nuevo registro: Chile: VII Región: Sector La Mina, Paso Internacional Pehuenche.

\section{Dargida polygona (Hampson)}

Tmetolophota polygona Hampson, 1905: 471

Dargida polygona (Hampson). Poole, 1989: 307

Localidad tipo: Perú: Huancabamba.

\section{Dargida procinctus (Grote)}

Eupsephopaectes procinctus Grote, 1873: 138

Dargida procinctus (Grote). Poole, 1989: 307

Localidad tipo: USA: California

43. Dargida quadrannulata (Morrison) n. comb. Mamestra quadrannulata Morrison, 1875: 430 Faronta quadrannulata (Morrison). Poole, 1989: 448

Localidad tipo: USA: Nebraska, Dodge Co., Glencoe.

\section{Dargida resputa (Draudt)}

Hadena resputa Draudt, 1924: 114

Dargida resputa (Draudt). Poole, 1989: 307

Localidad tipo: Colombia: Monte Tolima.

45. Dargida rubripennis (Grote \& Robinson) n. comb.

Leucania rubripennis Grote \& Robinson, 1870: 179

Faronta rubripennis (Grote \& Robinson). Franclemont, 1951: 74; Poole, 1989: 448

Localidad tipo: USA: Texas.

\section{Dargida scripta (Maassen)}

Mamestra scripta Maassen, 1890: 141, sensu Poole, 1989: 307

Strigania scripta (Maassen). Hampson, 1905: 467. Draudt in Seitz, 1927: 162

Dargida scripta (Maasen). Poole, 1989: 307

Localidad tipo: Bolivia: cerca de Tocora.

\section{Dargida semilunata Angulo \& Olivares}

Dargida semilunata Angulo \& Olivares. Rodríguez et al. 2001

Localidad tipo: Ecuador: Imbabura.

48. Dargida terrapictalis (Buckett) n. comb.

Faronta terrapictalis Buckett [1969] 1967: 268. Poole, 1989: 448.

Localidad tipo: USA: California, Modoc County, Alturas. 
49. Dargida tetera (Smith) n. comb.

Leucania tetera Smith, 1902:187

Faronta tetera (Smith). Poole, 1989: 448

Localidad tipo: USA: Arizona, Cochise Co., Wilgus.

\section{Dargida tridens (Köhler)}

Sideridis tridens Köhler, 1947: 93

Dargida tridens (Köhler). Poole, 1989: 307.

Localidad tipo: Argentina: Salta, Garrapatal.

\section{Dargida uncifera (Maassen)}

Prodenia uncifera Maassen in Weyner y Maassen, 1890: 140.

Dargida uncifera (Maassen). Poole, 1989: 307

Localidad tipo: Colombia: Chiles. Ecuador: Pichincha; Mojanda.

\section{Dargida uncisigna (Hampson)}

Hadena uncisigna Hampson, 1913: 593

Dargida uncisigna (Hampson). Poole, 1989: 307.

Localidad tipo: Perú: Acopampa.

\section{Dargida violascens (Maassen)}

Xylina violascens Maassen, 1890: 146, sensu Poole, 1989: 307

Dargida violascens (Maassen). Poole, 1989: 307. Localidad tipo: Ecuador: Páramo de Alao.

\section{DISCUSION}

Este estudio es insuficiente para dilucidar si el total de las especies incluídas en el presente catálogo crítico nominal de las especies de Dargida pertenecen a éste, ya que no han sido analizadas todas las 53 especies, lo que deberá esperar un estudio posterior.

La especie Strigania ecuatoriana Angulo \& Olivares (Angulo et al. 1999) no se incluye en este listado debido a que presenta el clásper desarrollado, lo que la margina de este grupo; esta especie pertenece al género Euryclasper Angulo \& Olivares (en prensa), por sus características, sin embargo, esto deberá ser estudiado más a fondo en forma posterior.

Dentro del catálogo crítico nominal de especies de Dargida, entregado en este trabajo, se encuentra un problema entre dos de sus especies. La especie $D$. albilinea presenta entre sus sinónimos a $D$. diffusa, según Parra et. al. (1986), sin embargo el catálogo de Poole 1989 presenta a ambas especies como válidas; este aspecto merece atención dado que este autor no considera el trabajo de Parra et al. (1986) en la elaboración de su catálogo.

De la sinonimia de Strigania y Dargida surge también que el carácter dígitus bífido, usados en la clave de diferenciación entre Scriptania, Strigania y Dargida por Angulo et al. (1999), para la separación de estos géneros y Scriptania, es sólo un carácter específico y no apoya una separación genérica.

Las diferencias de maculación advertidas por Franclemont (1951), entre los géneros Dargida y Faronta, son evidentes, y está basada en la falta de patrones cromáticos en las especies que pertenecían a Faronta, los que son abundantes en Dargida; sin embargo, la distribución geográfica de estos géneros puede explicar algo de estas diferencias, puesto que las especies de Faronta Smith (ahora Dargida) son de distribución Neártica y Neotropical (subregión andino-patagónica), y las especies originarias de Dargida de distribución Neotropical (subregión guyano-brasileña). Los diferentes hábitat zoogeográficos que encierran éstos, se corresponden con las manifestaciones cromáticas que presentan las especies que los habitan, siendo los más coloridos los guyanobrasileños ante los neárticos y los andinopatagónicos.

Las especies catalogadas para el género Dargida Walker están distribuídas en dos regiones zoogeográficas, la Región Neotropical y la Región Neártica, mostrando un continuo geográfico desde Canadá hasta Chile. El mayor número de sus especies está concentrado en la subregión guyanobrasileña (Fittkau 1968) de la Región Neotropical. No existen datos altitudinales de las especies de Dargida debido a que el material de terreno es escaso, sólo se conocen mayores antecedentes de especies de amplia distribución en Chile como $D$. albilinea y D. lithophilus, además de especies con localidades en el Paso Internacional Pehuenche para $D$. permira y de Ecuador, Imbabura, D. imitata, D. grammivora y $D$. semilunata, que vuelan por sobre los $2.000 \mathrm{msnm}$. De las especies restantes se desconocen datos. Dos de las especies listadas son consideradas de importancia económica para Chile. Dargida albilinea ha sido listada dentro de los Noctuidae de importancia agrícola (Parra et al. 1986). Tanto 
D. albilinea como Dargida lithophilus integran un listado de 29 especies asociadas a cultivos en Chile, la primera presente en cultivos de arroz, avena, trigo y remolacha, y la segunda presente en cultivos de trigo (Angulo et al. 1990).

\section{CONCLUSIONES}

1. El género Dargida Walker queda formado por 53 especies nominales, las que se listan en el catálogo crítico realizado en este estudio.

2. El género Strigania Hampson 1905 es sinónimo junior de Dargida Walker 1856, por principio de prioridad.

3. El género Dargida Walker pertenece al primer grupo de especies con corona uniseriada, caracterizado por Franclemont 1951 para los géneros afines a Leucania.

4. De las exclusiones e inclusiones de especies en el género Dargida, destaca:

Strigania ecuatoriana, que no es incluida en Dargida, debido a que presenta el clásper desarrollado, probablemente pertenezca al género Euryclasper Angulo \& Olivares. Falta una revisión taxonómica del género Dargida, que abarque a cada una de las especies listadas en él, pues pueden existir elementos extraños que pudieran ser segregados de éste.

5. Con respecto a la distribución geográfica del género Dargida, ésta se presenta como un continuo entre la región Neártica y Neotropical, siendo más representativa en esta última.

6. De la historia natural del género Dargida, sólo se tienen antecedentes para Chile de dos especies: Dargida albilinea y Dargida lithophilus, de las cuales se conocen sus estados inmaduros y su asociación a ciertos cultivos, por lo que se encuentran citadas para Chile como plagas de importancia agrícola, ambas de amplia distribución en el país.

\section{AGRADECIMIENTOS}

Se agradece a la Dirección de Investigación de la Universidad de Concepción por el apoyo prestado para el desarrollo de este trabajo, mediante el proyecto DIUC 203.113.060-1.0

\section{BIBLIOGRAFIA}

Angulo, A.O., C. Jana-Sáenz, L. E. Parra \& E. E. CAstillo. 1990. Lista de lepidópteros asociados a algunos cultivos en Chile (Lepidoptera: Noctuidae). Status sistemático actual. Gayana Zool. 54 (1-2): 51-61.

Angulo, A.O. \& T.S. Olivares. 1999. Nuevo género y nuevas especies de nóctuidos altoandinos II (Lepidoptera: Noctuidae). Gayana 63 (2): 1-10.

Angulo, A.O., M. Rodríguez \& T. S. Olivares. 1999. Nuevo género y nuevas especies de nóctuidos altoandinos III. Faronta Smith, 1908 v/s Strigania Hampson, 1905 y algunos alcances acerca de Dargida Walker, 1856 (Lepidoptera: Noctuidae). Gayana 63 (2): 67-85

Angulo, A.O. \& G. Th. Weigert. 1977. Pseudaletia punctulata (Blanchard) y Pseudaletia impuncta (Guenée): nóctuidos hadeninos similares en Chile. Lepidoptera: Noctuidae). AgroSur 5(1): 12-17.

BuCKETT, J. S. 1969. Revision of the Neartic moth genus Abagrotis Smith with descriptions of new species (Lepidoptera: Noctuidae), Part 5. Occasional Paper of the Bureau of Entomology of California. 17: $27 \mathrm{pp}$

ButLER, A.G. 1882. Heterocerous Lepidoptera collected in Chili by Thomas Edmonds, Esq. Trans. Ent. Soc. London. pp 114-116.

Dognin, P. 1911. Hétérocéres nouveau de l' Amerique du Sud. Fascicule III. Rennes. Oberthür. 66pp.

Draudt, M. 1924 -1927. In: Seitz, A. Die GrossSchmetterlinge des americanischen Faunengebietes. Eulenartige Nachtfalter (The Macrolepidoptera of the American region. Noctuiformes). 7 : 21-180.

DruCE, H. 1905. Description of some new species of Noctuidae from Tropical South America. Annals and Magazine of Natural History, series 7, 15: 372375.

Druce, H. 1908. Descriptions of some new species of Noctuidae from Peru. Annals and Magazine of Natural History, series 8, 1: 289-303.

Druce, H. 1911. Descriptions of some new species of Heterocera, mostly from Tropical South America. Annals and Magazine of Natural History, series 8, 8: 716-720.

DyaR, H.G. 1920 [1919]. New moths from Mexico (Lepidoptera, Noctuidae, Hadeninae). Insecutor Inscitiae Menstruus.7: 162-164.

DrucE, H. 1898. Heterocera. In: Godman, F.D. \& O. Salvin. Biologia Centrali-Americana; or Contributions to the knowledge of the fauna of Mexico and Central America. Zoology. Lepidoptera. London. Taylor and Francis. 1: 490 pp. Plates 1-64.

FittKau, E. J. 1968. [1974] La Fauna de Sudamérica. (Traducción de Alberto Larrain). Publicación especial. Sociedad de Biología de Concepción. Chile. $31 \mathrm{p}$.

ForBES, W.T.M. 1954. Lepidoptera of New York and neighboring states. Noctuidae. Part. III. Memoirs 329:433 pp. 
Franclemont, J.G. 1951. The species of the Leucania unipuncta group, with a discussion of the generic names for the various segregates of Leucania in North America (Lepidoptera: Phalaenidae: Hadeninae). Proc. Ent. Soc. of Washington 53(2): 57-85.

GoDFrey, G.L. 1972. A review and reclasification of larvae of the subfamily Hadeninae (Lepidoptera: Noctuidae) of America North of Mexico. USDA. Tech. Bull. 1450: 256 pp.

Grote, A. R. 1873. Description of North American Noctuidae. Bulletin of the Buffalo Society of the Natural Sciences. 1:190-194.

Grote, A. R. \& C. T. Robinson. 1870. Descriptions of American Lepidoptera, $\mathrm{N}^{\circ}$ 5. Transactions of the American Entomological Society. 1:323-360.

Hampson, G.F. 1905. Catalogue of the Lepidoptera Phalaenae in the British Museum. London. Taylor \& Francis. 5: 634 pp.

Hampson, G.F. 1909. Catalogue of the Lepidoptera Phalaenae in the British Museum. London. Taylor \& Francis. 9:552 pp.

Hampson, G.F. 1913. Catalogue of the Lepidoptera Phalaenae in the British Museum. London. Taylor \& Francis. 13: 609 pp.

HERRICH- SCHÄFFER, G.A.W. 1869. Sammlung neuer oder wenig Bekannter Aussereuropäischer Schmetterlinge, series 1. Regensburg.G.J. Manz. 2: 1-4. Plates 97-100, figs.552-571.

Huebner, J. 1826-1832. Zuträge zur Sammlung Exotischer Schmetterlinge. Augsburg. No publisher given. 4:48 pp. Plates 104-137.

KöHLER, P. 1947. Los “Noctuidae” Argentinos. Subfamilia Hadeninae. Acta Zoologica Lilloana. 12: 135-182.

KöHLER, P. 1959. Noctuidarum Miscellanea II (Lep. Noct.) Rev. Soc. Ent. Argentina 21 (3-4): 99-120.

KöHLER, P. 1968. Beiträge zur kenntnis der Insektenfauna Boliviens XXI. Lepidoptera IV. Noctuidae aus Bolivien (Heterocera). Veröffentlichungen der Zoologischen Staatssammlung München. 12: 319, 6 plates.

KöHLER, P. 1973. Noctuidarum Miscellanea V. (Lep. Het.). Acta Zoologica Lilloana 30: 13-21.

Weymer, G. \& P. Maasen. 1890. Lepidopteren gesammelt auf Einer Reise durch Colombia, Ec- uador, Peru, Brasilien, Argentinien und Bolivien in den Jarhren 1868-1877 von Alphons Stübel. Berlin. Asher and Co. 182 pp.

McDunnough, J.H. 1937a. Notes on North America noctuid genera. Canadian Entomologist 69: 40-47.

McDunnough, J.H. 1937b. A Correction. Canadian Entomologist 69: 141 .

Morrison, H.K. 1875. Notes on the Noctuidae with descriptions of certain new species. $\mathrm{N}^{\circ} 2$. Proceedings of the Academy of Natural Sciences of Philadelphia. 27: 428-436.

Parra, L. E. \& A.O. Angulo. 1988. Faronta albilinea (Hübner) y Faronta atrifera (Hampson) n. comb.: mariposas nocturnas similares (Lepidoptera: Noctuidae: Hadeninae). Gayana Zool. 52 (3-4): 261-270.

Parra, L.E., A.O. Angulo \& C. Jana-Sáenz. 1986. Lepidópteros de importancia agrícola: Clave práctica para su reconocimiento en Chile (Lepidoptera. Noctuidae). Gayana Zool. 50 (1-4): 81116.

Poole, R.W. 1989. Lepidopterorum Catalogus (N.S.) Noctuidae. Part. 1-2. E.J. Brill|Flora \& Fauna Publications. 118: 1013 pp.

Rodríguez, M. A.; T. S. Olivares \& A. O. Angulo. 2001. Nuevas especies altoandinas VII (Lepidoptera. Noctuidae). Rev. Biología Tropical. 49 (1): 317328.

Schaus, W. 1894. New species of Noctuidae from Tropical America. Transactions of the American Entomological Society. 21: 223-224.

Sмiтh, J. B. 1902. New species of Noctuidae for 1902. Journal of the New York Entomological Society. 10: 33-53.

Sмiтh, J.B. 1908. New species and genera of the Lepidopterous family Noctuidae for 1907 (Part II). Annals of the New York Academy of Sciences. 18: 91-127.

WALKER, F. 1856. List of the specimens of Lepidopterous insects in the collection of the British $\mathrm{Mu}$ seum 10: 334 .

Zerny, H. 1916. Neue Heterocerean aus der Sammlung des k. k. Naturhistorischen Hofmuseum in Wien. Annalen des k.k. Naturhistorischen Hofmuseum. 30: 173-195. 